\title{
Effective transvascular delivery of nanoparticles across the blood-brain tumor barrier into malignant glioma cells
} Hemant Sarin*1,2, Ariel S Kanevsky², Haitao $\mathrm{Wu}^{3}$, Kyle R Brimacombe ${ }^{4}$, Steve H Fung 5 , Alioscka A Sousa ${ }^{1}$, Sungyoung Auh ${ }^{6}$, Colin M Wilson ${ }^{3}$, Kamal Sharma7,8, Maria A Aronova1, Richard D Leapman¹, Gary L Griffiths ${ }^{3}$ and Matthew D Hall ${ }^{4}$

\begin{abstract}
Address: ${ }^{1}$ National Institute of Biomedical Imaging and Bioengineering, National Institutes of Health, Bethesda, Maryland 20892, USA, ${ }^{2}$ Diagnostic Radiology Department, Clinical Center, National Institutes of Health, Bethesda, Maryland 20892, USA, ${ }^{3}$ Imaging Probe Development Center, National Heart, Lung, and Blood Institute, National Institutes of Health, Bethesda, Maryland 20892, USA, ${ }^{4}$ Laboratory of Cell Biology, National Cancer Institute, National Institutes of Health, Bethesda, Maryland 20892, USA, ${ }^{5}$ Neuroradiology Department, Massachusetts General Hospital, Boston, Massachusetts 02114, USA, ${ }^{6}$ Biostatistics, National Institute of Neurological Disorders and Stroke, National Institutes of Health, Bethesda, Maryland 20892, USA, ${ }^{7}$ Metabolism Branch, National Cancer Institute, National Institutes of Health, Bethesda, Maryland 20892, USA and ${ }^{8}$ Division of Biologic Drug Products, Office of Oncology Products, Center for Drug Evaluation and Research, U.S. Food \& Drug Administration, Silver Spring, Maryland 20993, USA
\end{abstract}

Email: Hemant Sarin* - sarinh@mail.nih.gov; Ariel S Kanevsky - kanevskya@mail.nih.gov; Haitao Wu - wuh3@mail.nih.gov; Kyle R Brimacombe - brimacombek@mail.nih.gov; Steve H Fung - sfung@partners.org; Alioscka A Sousa - sousaali@mail.nih.gov; Sungyoung Auh - auhs@mail.nih.gov; Colin M Wilson - wilsoncm@mail.nih.gov; Kamal Sharma - kamal.sharma@fda.hhs.gov; Maria A Aronova - aronovaa@mail.nih.gov; Richard D Leapman - leapmanr@mail.nih.gov; Gary L Griffiths - griffithsgl@mail.nih.gov; Matthew D Hall - hallma@mail.nih.gov

* Corresponding author

Published: 18 December 2008

Journal of Translational Medicine 2008, 6:80 doi:10.1 I86/1479-5876-6-80
Received: 20 October 2008

Accepted: 18 December 2008

This article is available from: http://www.translational-medicine.com/content/6/I/80

(c) 2008 Sarin et al; licensee BioMed Central Ltd.

This is an Open Access article distributed under the terms of the Creative Commons Attribution License (http://creativecommons.org/licenses/by/2.0), which permits unrestricted use, distribution, and reproduction in any medium, provided the original work is properly cited.

\begin{abstract}
Background: Effective transvascular delivery of nanoparticle-based chemotherapeutics across the blood-brain tumor barrier of malignant gliomas remains a challenge. This is due to our limited understanding of nanoparticle properties in relation to the physiologic size of pores within the blood-brain tumor barrier. Polyamidoamine dendrimers are particularly small multigenerational nanoparticles with uniform sizes within each generation. Dendrimer sizes increase by only I to 2 $\mathrm{nm}$ with each successive generation. Using functionalized polyamidoamine dendrimer generations I through 8 , we investigated how nanoparticle size influences particle accumulation within malignant glioma cells.
\end{abstract}

Methods: Magnetic resonance and fluorescence imaging probes were conjugated to the dendrimer terminal amines. Functionalized dendrimers were administered intravenously to rodents with orthotopically grown malignant gliomas. Transvascular transport and accumulation of the nanoparticles in brain tumor tissue was measured in vivo with dynamic contrast-enhanced magnetic resonance imaging. Localization of the nanoparticles within glioma cells was confirmed ex vivo with fluorescence imaging.

Results: We found that the intravenously administered functionalized dendrimers less than approximately II.7 to $11.9 \mathrm{~nm}$ in diameter were able to traverse pores of the blood-brain tumor barrier of RG-2 malignant gliomas, while larger ones could not. Of the permeable functionalized 
dendrimer generations, those that possessed long blood half-lives could accumulate within glioma cells.

Conclusion: The therapeutically relevant upper limit of blood-brain tumor barrier pore size is approximately II.7 to $11.9 \mathrm{~nm}$. Therefore, effective transvascular drug delivery into malignant glioma cells can be accomplished by using nanoparticles that are smaller than II.7 to II.9 $\mathrm{nm}$ in diameter and possess long blood half-lives.

\section{Background}

Progress towards the effective clinical treatment of malignant gliomas has been hampered due to ineffective drug delivery across the blood-brain tumor barrier (BBTB), in addition to the inability to simultaneously image drug permeation through tumor tissue [1-3]. The current paradigm for treating malignant gliomas is the placement of implantable 1,3-bis (2-chloroethyl)-1-nitrosourea (BCNU, also called carmustine) wafers in the tumor resection cavity followed by administration of oral temozolomide, an alkylating agent, with concurrent radiation [4-7]. $\mathrm{BCNU}$, a low molecular weight nitrosourea, is able to cross the BBTB, but is unable to accumulate within malignant glioma cells at therapeutic levels due to a short blood half-life [8]. Intra-operative placement of polymeric wafers impregnated with BCNU along the tumor resection cavity has resulted in improved patient outcomes, and significantly decreased toxicity compared to that associated with intravenous BCNU treatment $[9,10]$. Since this local method of BCNU delivery circumvents the BBTB and allows for sustained release of BCNU from the polymer, there are higher steady-state BCNU concentrations within the tumor resection cavity[11]. However, a major limitation of this delivery method is that the placement of the BCNU polymer wafers may only be performed at the time of initial tumor resection [12]. Temozolomide, like BCNU, has a low molecular weight and a short blood half-life which limits its ability to accumulate within malignant glioma cells $[5,13]$.

The sizes of traditional chemotherapeutics, such as BCNU and temozolomide, are commonly reported as particle molecular weights since these particles are usually smaller than $1 \mathrm{~nm}$ in diameter [13]. In contrast, the sizes of nanoparticle-based therapeutics are commonly reported as particle diameters since these particles usually range between 1 and $200 \mathrm{~nm}$ in diameter [14,15]. Particle shapes and sizes determine how effectively particles can be filtered by the kidneys [16-18]. Spherical nanoparticles smaller than 5 to $6 \mathrm{~nm}$ and weighing less than 30 to $40 \mathrm{kD}$ are efficiently filtered by the kidneys [17]. Spherical nanoparticles that are larger and heavier are not efficiently filtered by the kidneys; therefore, these particles possess longer blood half-lives [19]. The BBTB of malignant gliomas becomes porous due to the formation of discontinu- ities within and between endothelial cells lining the lumens of tumor microvessels [20]. Nanoparticles smaller than the pores within the BBTB, with long blood halflives, could function as effective transvascular drug delivery devices for the sustained-release of chemotherapeutics into malignant glioma cells.

Even though fenestrations and gaps within the BBTB of malignant gliomas allow for unimpeded passage of low molecular weight therapeutics [21], these pores are narrow enough to prevent the effective transvascular passage of most nanoparticles [22-25]. If the upper limit of the therapeutically relevant pore size of the BBTB could be accurately determined, then intravenously administered nanoparticles, with long blood half-lives, could serve as effective drug delivery vehicles across the BBTB of malignant gliomas.

By performing intravital fluorescence microscopy of xenografted human glioma microvasculature in the mouse cranial window model, Hobbs et al. [26] observed perivascular fluorescence 24 hours following the intravenous infusion of rhodamine dye labeled liposomes of 100 nm diameters. Since then several classes of nanoparticles have been designed to be less than $100 \mathrm{~nm}$ in diameter for the purposes of effective transvascular drug delivery across the BBTB. These classes of nanoparticles include metalbased (i.e. iron oxide) [27], lipid-based (i.e. liposomes) [28], and biological-based (i.e. antibodies, viruses) $[29,30]$.

Yet another class of nanoparticles are the polymer-based dendrimers [2,31]. Polyamidoamine (PAMAM) dendrimers [32] are multigenerational polymers with a branched exterior consisting of surface groups that can be functionalized with imaging [33,34], targeting [35], and therapeutic agents $[35,36]$. PAMAM dendrimers functionalized with low molecular weight agents remain particularly small, typically ranging between $1.5 \mathrm{~nm}$ (generation 1 , G1) and $14 \mathrm{~nm}$ in diameter (generation 8, G8) [32,33]. Particle shapes are spherical and sizes are uniform within a particular generation. With each successive dendrimer generation, the number of modifiable surface groups doubles while the overall diameter increases by only 1 to $2 \mathrm{~nm}$ [37]. 
We hypothesized that the major reason for the ineffectiveness of metal-based, lipid-based and biological-based nanoparticles in traversing the BBTB of malignant gliomas is the large size of these particles relative to the physiologic pore size of the BBTB. In this work, using the RG-2 malignant glioma model $[38,39]$, we also investigated how the transvascular transport of dendrimer nanoparticles is affected by tumor volume-related differences in the degree of BBTB breakdown.

The hyperpermeability of the BBTB of malignant gliomas results in contrast enhancement of brain tumor tissue on magnetic resonance imaging (MRI) scans following the intravenous infusion of gadolinium (Gd)-diethyltriaminepentaacetic acid (DTPA), a low molecular weight contrast agent $[40,41]$. To visualize the extravasation of PAMAM dendrimers across the BBTB of rodent malignant gliomas by dynamic contrast-enhanced MRI, we functionalized the exterior of PAMAM dendrimers with Gd-DTPA. Using dynamic contrast-enhanced MRI, we measured the change in contrast enhancement of malignant gliomas for up to 2 hours following the intravenous infusion of successively higher Gd-dendrimer generations up to, and including, Gd-G8 dendrimers. To verify that dendrimer size, and not dendrimer generation, is the primary determinant of particle blood half-life, we studied Gd-G4 dendrimers of two different sizes. One was a lowly conjugated Gd-G4 weighing $24.4 \mathrm{kD}$ and the other was a standard Gd-G4 weighing $39.8 \mathrm{kD}$. The Gd concentration, a surrogate for the amount of Gd-dendrimer within tumor tissue, was determined by measuring the molar relaxivity of Gddendrimers in vitro in combination with the change in the blood and tissue longitudinal relaxivities $\left(\mathrm{T}_{1}\right)$ before and after Gd-dendrimer infusion [42]. Based on comparisons of the contrast enhancement patterns of malignant gliomas for up to 2 hours, within a particular Gd-dendrimer generation as well as across Gd-dendrimer generations, we determined the physiologic upper limit of BBTB pore size.

In addition to the in vivo dynamic contrast-enhanced MRI experiments with Gd-dendrimers, we performed in vitro and ex vivo fluorescence microscopy experiments using rhodamine B labeled Gd- dendrimers to confirm that the impediment to the cellular uptake of functionalized dendrimers is the BBTB. The observations made in this study, using functionalized dendrimers, are to serve as a guide for designing nanoparticles that are effective at traversing the pores of the blood-brain tumor barrier and accumulating within individual glioma cells.

\section{Methods}

PAMAM dendrimer functionalization and characterization Bifunctional chelating agents and gadolinium-benzyldiethyltriaminepentaacetic acid (Gd-Bz-DTPA) function- alized PAMAM dendrimers were synthesized according to described procedures with minor modifications, as were the corresponding rhodamine-substituted conjugates [4345]. Gd-dendrimers, with the exception of lowly conjugated Gd-G4, were prepared by using a molar reactant ratio of $\geq 2: 1$ bifunctional chelate to dendrimer surface amine groups. For lowly conjugated Gd-G4 a lower molar reactant ratio of 1.1:1 was used to limit conjugation. The duration of the chelation reaction for the lowly conjugated Gd-G4 was 24 hours as compared to the standard 48 hours for chelation of all other dendrimers. Rhodamine B labeled Gd-dendrimers were prepared by stirring rhodamine $\mathrm{B}$ isothiocyanate (RBITC) and PAMAM dendrimers at a 1:9 molar ratio of RBITC to dendrimer surface amine groups in methanol at room temperature for 12 hours. Isothiocyanate activated DTPA was then added in excess and reacted for an additional 48 hours. Gadolinium was then chelated after the removal of the $t$-butyl protective groups on DTPA. The percent by mass of Gd in each Gddendrimer generation was determined by elemental analysis to be: Gd-G1 (15.0\%), Gd-G2 (14.8\%), Gd-G3 $(12.9 \%)$, lowly conjugated Gd-G4 (12.3\%), standard GdG4 (12.0\%), Gd-G5 (11.9\%), Gd-G6 (11.9\%), Gd-G7 (12.2\%), Gd-G8 (10.2\%). The Gd percent by mass for the rhodamine B Gd-dendrimers was determined to be: rhodamine B Gd-G2 (9.6\%), rhodamine B Gd-G5 (9.8\%), rhodamine B Gd-G8 (9.3\%). Gd-G1 through Gd-G5 dendrimer molecular weights were determined by matrix assisted laser desorption/ionization time-of-flight (MALDI-TOF) mass spectroscopy (Scripps Center for Mass Spectrometry, La Jolla, CA). Gd percent by mass of the Gd-dendrimer, in its solid form, was determined with the inductively coupled plasma-atomic emission spectroscopy (ICP-AES) method (Desert Analytics, Tucson, AZ). Gd-dendrimer infusions were normalized to $100 \mathrm{mM}$ with respect to $\mathrm{Gd}$, while rhodamine $\mathrm{B}$ Gd-dendrimer infusions were normalized to $67 \mathrm{mM}$ with respect to $\mathrm{Gd}$, in order to guarantee proper solvation.

\section{In vitro scanning transmission electron microscopy}

For in vitro transmission electron microscopy experiments, a $5 \mu$ d droplet of phosphate-buffer saline solution containing a sample of Gd-dendrimers from generations $5,6,7$ or 8 was absorbed onto a 3 nm-thick carbon support film covering the copper electron microscopy grids. Lacey Formvar/carbon coated 300 meshcopper grids supporting an ultrathin $3 \mathrm{~nm}$ evaporated carbon film were glow-discharged an air pressure of 0.2 mbar to facilitate Gd-dendrimer adsorption. After adsorption for 2 minutes, excess Gd-dendrimer solution was blotted with filter paper. The grids were then washed 5 times with $5 \mu \mathrm{L}$ aliquots of deionized water, and left to dry in air. Annular dark field scanning transmission electron microscope (ADF STEM) images of the Gd-dendrimers were recorded using a Tecnai TF30 electron microscope (FEI, Hillsboro, 
OR, USA) equipped with a Schottky field-emission gun and an in-column ADF detector (Fischione, Export, PA) [46].

\section{In vitro fluorescence experiments}

For in vitro fluorescence experiments, RG-2 glioma cells were plated on Fisher Premium coverslips (Fisher Scientific, Pittsburgh, PA) and incubated in wells containing sterile $3 \mathrm{ml}$ DME supplemented with 10\% FBS (Invitrogen, Carlsbad, CA). The RG-2 glioma colonies were allowed to establish for 24 hours in an incubator set at $37^{\circ} \mathrm{C}$ and $5 \% \mathrm{CO}_{2}$. Rhodamine $\mathrm{B}$ Gd-G2, rhodamine $\mathrm{B}$ Gd-G5 or rhodamine B Gd-G8 dendrimers were added to the medium by equivalent molar rhodamine B concentrations of $7.2 \mu \mathrm{M}$ and the cells were incubated in the dark for another 4 hours. Following incubation, cells were washed 3 times with PBS, then $50 \mu$ DAPI-Vectashield nuclear stain medium (Vector Laboratories, Burlingame, CA) was placed on the coverslips for 15 minutes. Coverslips were then inverted and mounted on Daigger Superfrost slides (Daigger, Vernon Hills, IL) and sealed into place. Confocal imaging was performed on a Zeiss 510 NLO microscope (Carl Zeiss MicroImaging, Thornwood, NY). Slides were stored in the dark while not being analyzed.

\section{In vitro magnetic resonance imaging for calculations of Gd-dendrimer molar relaxivity}

Gd-dendrimer stock solution $(20 \mu \mathrm{l}$ of $100 \mathrm{mM})$ and rhodamine B Gd-dendrimer stock solution (30 $\mu$ l of 67 $\mathrm{mM}$ ) for the particular generation, used for in vivo imaging, was diluted using PBS into $200 \mu \mathrm{l}$ microfuge tubes at $0.00 \mathrm{mM}, 0.25 \mathrm{mM}, 0.50 \mathrm{mM}, 0.75 \mathrm{mM}$ and $1.00 \mathrm{mM}$ with respect to $\mathrm{Gd}$. As an external control, Magnevist (Bayer, Toronto, Canada), a form of Gd-DTPA, was also diluted at the above concentrations into $200 \mu \mathrm{l}$ microfuge tubes. The microfuge tubes were secured in level and upright positions within a plastic container filled with deionized ultra pure water. The container was placed in a $7 \mathrm{~cm}$ small animal solenoid radiofrequency coil (Philips Research Laboratories, Hamburg, Germany) centered within a 3.0 Tesla MRI scanner (Philips Intera; Philips Medical Systems, Andover, MA). Gd signal intensity measurements were made using a series of $\mathrm{T}_{1}$ weighted spin echo sequences with identical $\mathrm{T}_{\mathrm{E}}$ (echo time, $10 \mathrm{~ms}$ ) but different $\mathrm{T}_{\mathrm{R}}$ (repetition time, $100 \mathrm{~ms}, 300 \mathrm{~ms}, 600 \mathrm{~ms}$ and $1200 \mathrm{~ms}$ ). Using the measured Gd signal intensity, in addition to the known values for $T_{R}$ and $T_{E}$, the $T_{1}$ and equilibrium magnetization $\left(\mathrm{M}_{0}\right)$ were calculated by nonlinear regression [42]. In vitro and in vivo Gd-dendrimer molar relaxivities were assumed to be equivalent for the purposes of this work.

\section{Brain tumor induction and animal preparation for imaging} All animal experiments were approved by the National Institutes of Health Clinical Center Animal Care and Use Committee. Cryofrozen pathogen-free RG-2 glioma cells were obtained from the American Type Culture Collection (Rockville, MD) and cultured in sterile DME supplemented with 10\% FBS and 2\% penicillin-streptomycin in an incubator set at $37^{\circ} \mathrm{C}$ and $5 \% \mathrm{CO}_{2}$. The anesthesia and route for all animal experiments was isoflurane by inhalation with nose cone, $5 \%$ for induction and 1 to $2 \%$ for maintenance. On experimental day 0 , the head of anesthetized adult male Fischer344 rats (F344) weighing 200250 grams (Harlan Laboratories, Indianapolis, IN) was secured in a stereotactic frame with ear bars (David Kopf Instruments, Tujunga, CA). The right anterior caudate and left posterior thalamus locations within the brain were stereotactically inoculated with RG-2 glioma cells [47]. In each location, either 20,000 or 100,000 glioma cells in 5 $\mu \mathrm{l}$ of sterile PBS were injected over 8 minutes, using a 10 $\mu$ Hamilton syringe with a 32-gauge needle. With this approach the majority of animal brains developed one large and one small glioma. On experimental days 11 to 12 , brain imaging of re-anesthetized rats was performed following placement of polyethylene femoral venous and arterial cannulas (PE-50; Becton-Dickinson, Franklin Lakes, NJ), for contrast agent infusion and blood pressure monitoring, respectively. After venous cannula insertion, $50 \mu \mathrm{l}$ of blood was withdrawn from the venous cannula for measurement of hematocrit.

\section{In vivo magnetic resonance imaging of brain tumors}

All magnetic resonance imaging experiments were conducted with a 3.0 Tesla MRI scanner (Philips Intera) using a $7 \mathrm{~cm}$ solenoid radiofrequency coil (Philips Research Laboratories). For imaging, the animal was positioned supine, with face, head, and neck snugly inserted into a nose cone centered within the $7 \mathrm{~cm}$ small animal solenoid radiofrequency coil. Anchored to the exterior of the nose cone were three $200 \mu \mathrm{L}$ microfuge tubes containing 0.00 $\mathrm{mM}, 0.25 \mathrm{mM}$ and $0.50 \mathrm{mM}$ solutions of Magnevist to serve as standards for measurement of MRI signal drift over time. Fast spin echo $\mathrm{T}_{2}$ weighted anatomical scans were performed with $\mathrm{T}_{\mathrm{R}}=6000 \mathrm{~ms}$ and $\mathrm{T}_{\mathrm{E}}=70 \mathrm{~ms}$. Two different flip angle (FA) 3-D fast field echo (3D FFE) T1 weighted scans were performed with $\mathrm{T}_{\mathrm{R}}=8.1 \mathrm{~ms}$ and $\mathrm{T}_{\mathrm{E}}=$ $2.3 \mathrm{~ms}$, for quantification of $\mathrm{Gd}$ concentration. The first FFE scan was performed at a low FA of $3^{\circ}$ without any contrast agent on board. The second FFE scan was performed with a high FA of $12^{\circ}$. For this scan, the dynamic scan, each brain volume was acquired once every 20 seconds, for 1 to 2 hours. During the beginning of the dynamic scan, three to five baseline brain volumes were acquired prior to Gd-dendrimer infusion. Gd-dendrimers were infused at doses of $0.03,0.06$ or $0.09 \mathrm{mmol} \mathrm{Gd} / \mathrm{kg}$ bw depending on the experiment. Gd-dendrimer was 
infused as a bolus over 1 minute in order to accurately measure the contrast agent dynamics in blood during the bolus. Following completion of the 1 or 2 hour dynamic contrast-enhanced MRI scan, another 15 minute dynamic contrast-enhanced MRI scan was performed during which Magnevist was infused at a dose of $0.30 \mathrm{mmol} \mathrm{Gd} / \mathrm{kg}$ bw over 1 minute. Tumor regions of interest were drawn based on the Magnevist dynamic scan data.

\section{Dynamic contrast-enhanced MRI data analyses and pharmacokinetic modeling}

Imaging data was analyzed using the Analysis of Functional NeuroImaging (AFNI; http://afni.nimh.nih.gov/) software suite and its native file format [48]. Motion correction was performed by registering each volume of the dynamic high FA scan to its respective low FA scan. Alignments were performed using Fourier interpolation. A baseline $T_{1}$ without contrast $\left(T_{10}\right)$ map was generated by solving equation 1 (the steady-state for incoherent signal after neglecting $\mathrm{T}_{2}{ }^{*}$ effects) voxel-by-voxel for $\mathrm{T}_{1}$, at both low and high FA's, before contrast was infused [42].

$$
S=\frac{M_{0}\left(1-E_{1}\right) \sin q}{1-E_{1} \cos q}
$$

where

$$
E_{1}=\exp \left(-\frac{T_{R}}{T_{1}}\right)
$$

After determining the $\mathrm{T}_{10}$ value at each voxel, $\mathrm{T}_{1}$ map was calculated using equations 1 and 2 for each voxel of each dynamic image during the high FA scan after contrast infusion [42]. Datasets were converted to Gd concentration space [42]. Whole tumor regions of interest were drawn on the basis of the dynamic contrast enhancement pattern of tumor tissue observed following the infusion of Magnevist. These data were important for the drawing of accurate whole tumor regions of interest for minimally enhancing gliomas, especially for all malignant gliomas within the $0.03 \mathrm{mmol} \mathrm{Gd} / \mathrm{kg}$ bw Gd-dendrimer dose category and those in the $0.09 \mathrm{mmol} \mathrm{Gd} / \mathrm{kg}$ bw Gd-G8 dendrimer dose sub-category. Normal brain regions of interest were spherical $9 \mathrm{~mm}^{3}$ volumes in the left anterior caudate.

The pharmacokinetic properties of Gd-G1 through lowly conjugated Gd-G4 dendrimers were modeled using the dynamic contrast-enhanced MRI data from the groups of animals receiving $0.09 \mathrm{mmol} \mathrm{Gd} / \mathrm{kg}$ bw Gd-dendrimer infusions. The change in blood Gd-dendrimer concentration over time was obtained by selecting 2 to 3 voxels within the superior sagittal sinus, a large caliber vein that is minimally where influenced by in-flow and partial volume averaging effects. Since the transit time of blood movement between an artery and a vein within the brain is approximately 4 seconds, while the image acquisition rate was once every 20 seconds, the superior sagittal sinus was used for generation of the vascular input function for pharmacokinetic modeling [41]. Animal brains from which an optimal vascular input function could not be obtained were excluded from being analyzed by pharmacokinetic modeling. The voxels chosen had peak blood Gd concentrations closest to the calculated initial Gd-dendrimer volume of distribution, based on the blood volume of a 250 gram rat being $14 \mathrm{ml} \mathrm{[49].} \mathrm{Blood}$ concentration was converted to plasma concentration by correcting for the hematocrit (Hct) as shown in equation 3 [40].

$$
C_{\mathrm{p}}=\frac{C_{\mathrm{b}}}{1-\mathrm{Hct}}
$$

The 2-compartment 3-parameter generalized kinetic model (equation 4) $[40,50]$ was employed for pharmacokinetic modeling by performing voxel-by-voxel nonlinear regression over all time points.

$$
C_{\mathrm{t}}(t)=v_{\mathrm{p}} C_{\mathrm{p}}(t)+K^{\mathrm{trans}} \int_{0}^{t} C_{\mathrm{p}}(t) \exp \left(\frac{-K^{\text {trans }}(t-t)}{v_{\mathrm{e}}}\right) d t
$$

Constraints on the parameters were set between 0 and 1 calling on 10,000 iterations. Least squares minimizations were performed by implementing the Nelder-Mead simplex algorithm. Prior to statistical analysis, voxels with poor fits or non-physiologic parameters were censored.

\section{Ex vivo fluorescence microscopy and histological staining of brain tumor sections}

Six additional rats received $0.06 \mathrm{mmol} \mathrm{Gd} / \mathrm{kg}$ bw of rhodamine B Gd-G5 and two additional rats received 0.06 mmol Gd/kg bw of rhodamine B Gd-G8. Subsequent to the standard 2 hour dynamic contrast-enhanced MRI study, the brains of these animals were harvested and snap-frozen. On the day of cryosectioning, two $10 \mu \mathrm{m} \mathrm{sec-}$ tions of tumor bearing brain were cut onto each Daigger Superfrost slide with a Leica Cryotome (Leica, Bensheim, Germany). The first of two slides was prepared for fluorescence microscopy by application of DAPI-Vectashield nuclear stain medium and coversliping. Confocal imaging was performed on a Zeiss 510 NLO microscope. The second slide was stained with Hematoxylin and Eosin for visualization of tumor histology.

\section{Statistical analysis for pharmacokinetic modeling}

Vascular parameter pharmacokinetic values for individual tumor voxels were averaged in order to yield one value per parameter per tumor per rat, with tumors within a rat 
being treated as correlated. On the basis of the range of individual tumor volumes within Gd-G1, Gd-G2, Gd-G3 and lowly conjugated Gd-G4 dendrimer study groups, a dichotomous variable for tumor size was generated by using $50 \mathrm{~mm}^{3}$ as the cut-off between large and small tumors. Multivariate analysis of variance (MANOVA) models were used to examine the effect of dendrimer generation and tumor size. Prior to the MANOVA, it determined that there was no interaction between dendrimer generation and tumor size on any of the three parameters. The covariance structure was considered to be compound symmetric and the Kenward-Roger degrees of freedom method was used. Post-hoc comparisons between lowly conjugated Gd-G4 and each of the other generations were conducted. The significant $P$-values we report are following Bonferroni correction for multiple comparisons. Analyses were implemented in SAS PROC Mixed (SAS Institute Inc., Cary, North Carolina) with $\alpha=0.05$.

\section{Results}

\section{Physical properties of naked PAMAM and Gd-PAMAM}

\section{dendrimer generations}

The physical properties of naked PAMAM dendrimers (Starburst G1-G8, ethylenediamine core; Sigma-Aldrich, St. Louis, MO) and Gd-PAMAM dendrimers are detailed in table 1. Naked full generation PAMAM dendrimers are cationic due to the presence of amine groups on the dendrimer exterior for conjugation (Figure 1A). With each successive dendrimer generation both the molecular weight and number of terminal amines doubles. Conjugation of Gd-DTPA (charge -2, molecular weight $\sim 0.7 \mathrm{kD}$ ) to the surface amine groups of naked PAMAM dendrimers neutralizes the positive charge on dendrimer exterior (Figure $1 \mathrm{~B}$ ). The molecular weight increase of the naked dendrimer to that of the Gd-DTPA conjugated dendrimer is proportional to the percent conjugation of Gd-DTPA
(Table 1). The percent conjugation of lowly conjugated Gd-G4 dendrimers was $29.8 \%$ whereas that of standard Gd-G4 dendrimers was $47.5 \%$ (Table 1 ). The constants of proportionality required for calculation of Gd concentration, also known as Gd-dendrimer molar relaxivities, ranged between 7.8 and $12.2 \mathrm{~s} / \mathrm{mM}$ (Table 1 ).

Since the sizes of hydrated dendrimer generations, measured by small-angle X-ray scattering (SAXS) [51] and small-angle neutron scattering (SANS) [52], are similar to the sizes of respective dehydrated dendrimer generations measured by TEM [37], we were able to use ADF STEM to image Gd-G5 and higher generation Gd-dendrimers: these Gd-dendrimer generations possessed masses heavy enough to be visualized by ADF STEM $[46,53]$. ADF STEM images of Gd-G5 through Gd-G8 dendrimers demonstrated uniformity in particle size, shape and density within any particular dendrimer generation (Figure 1C). These images also confirmed a small increase of approximately $2 \mathrm{~nm}$ in particle diameter between successive generations. The diameters of sixty Gd-G7 and Gd-G8 dendrimers were measured. The average diameter of our Gd-G7 dendrimers was $11.0 \pm 0.7 \mathrm{~nm}$ and that of Gd-G8 dendrimers was $13.3 \pm 1.4 \mathrm{~nm}$ (mean \pm standard deviation).

\section{Effect of Gd-dendrimer dose on particle extravasation across the blood-brain tumor barrier}

The transvascular transport of Gd-G1 through Gd-G8 dendrimers across pores of the BBTB and accumulation within brain tumor tissue were studied at Gd-dendrimer doses of $0.03 \mathrm{mmol} \mathrm{Gd} / \mathrm{kg}$ bw and $0.09 \mathrm{mmol} \mathrm{Gd} / \mathrm{kg}$ bw. The $0.03 \mathrm{mmol} \mathrm{Gd} / \mathrm{kg}$ bw dose is the standard intravenous Gd-dendrimer dose for pre-clinical imaging with Gd-dendrimers [33]. For each Gd-dendrimer generation, the amount of Gd-dendrimer infused at the $0.03 \mathrm{mmol}$

Table I: Table I - Physical properties of PAMAM and Gd-PAMAM dendrimer generations

\begin{tabular}{|c|c|c|c|c|c|}
\hline $\begin{array}{l}\text { Dendrimer generation } \\
(\mathrm{G})\end{array}$ & No. terminal amines & $\begin{array}{c}\text { Naked PAMAM } \\
\text { molecular weight }{ }^{\#}(\mathrm{kD})\end{array}$ & $\begin{array}{l}\text { Gd-PAMAM molecular } \\
\text { weight }^{\dagger}(k D)\end{array}$ & $\begin{array}{c}\text { Gd-DTPA conjugation } \\
(\%)\end{array}$ & $\begin{array}{c}\text { Molar relaxivity } \\
(\mathrm{s} / \mathrm{mM})\end{array}$ \\
\hline GI & 8 & 1.43 & 5.63 & 67.1 & 9.8 \\
\hline G2 & 16 & 3.26 & 11.2 & 65.9 & 10.1 \\
\hline G3 & 32 & 6.91 & 18.6 & 47.7 & 10.4 \\
\hline $\begin{array}{c}\text { Lowly } \\
\text { conjugated } \\
\text { G4 }\end{array}$ & 64 & 14.2 & 24.4 & 29.8 & 7.8 \\
\hline $\begin{array}{l}\text { Standard } \\
\quad \text { G4 }\end{array}$ & 64 & 14.2 & 39.8 & 47.5 & 12.2 \\
\hline G5 & 128 & 28.8 & 79.8 & 47.2 & 10.9 \\
\hline G6 & 256 & 58.0 & 133 & 39.9 & 10.6 \\
\hline G7 & 512 & 116 & $330 \ddagger$ & 50.0 & 10.3 \\
\hline G8 & 1024 & 233 & $597 \ddagger$ & 37.8 & 9.4 \\
\hline
\end{tabular}

\#obtained from Dendritech, Inc.

tmeasured by MALDI-TOF MS unless noted otherwise

¥measured by ADF STEM

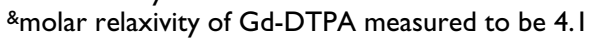



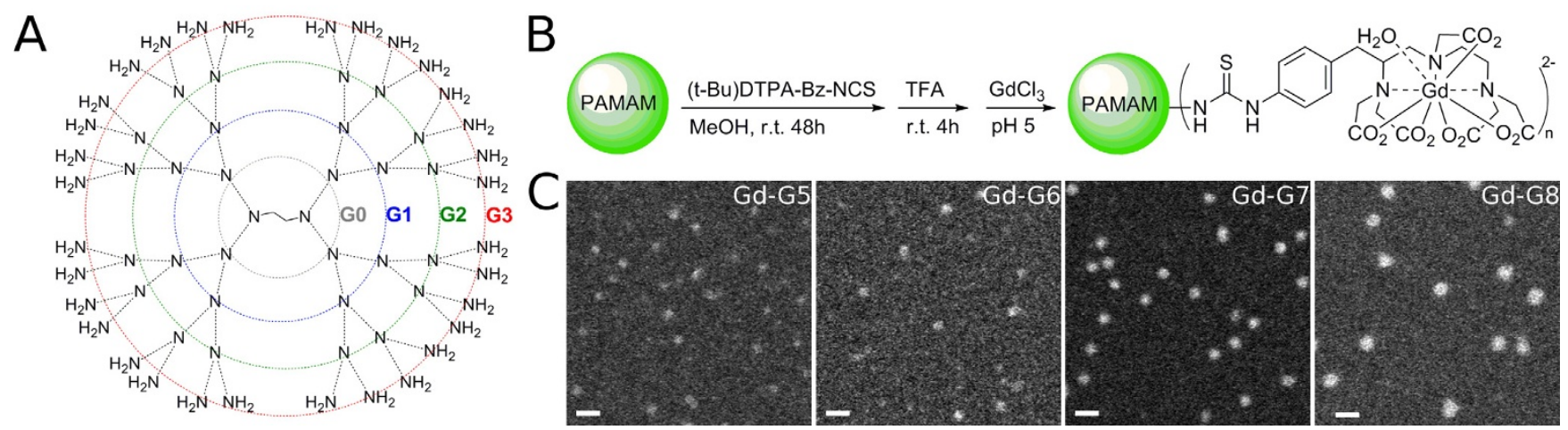

Figure I

Synthesis of Gd-dendrimers and transmission electron microscopy of higher generation Gd-dendrimers. A) A two-dimensional representation of naked polyamidoamine dendrimers up until generation 3 showing ethylenediamine core. B) The naked dendrimer has a cationic exterior. Functionalizing the terminal amine groups with Gd-diethyltriaminepentaacetic acid (charge -2) neutralizes the positive charge on the dendrimer exterior. C) Annular dark-field scanning transmission electron microscopy images of Gd-G5, Gd-G6, Gd-G7, and Gd-G8 dendrimers adsorbed onto an ultrathin carbon support film. Scale bar $=20 \mathrm{~nm}$.

$\mathrm{Gd} / \mathrm{kg}$ bw and $0.09 \mathrm{mmol} \mathrm{Gd} / \mathrm{kg}$ bw doses is shown in the supplementary table (Additional file 1).

At the $0.03 \mathrm{mmol} \mathrm{Gd} / \mathrm{kg}$ bw dose, Gd-G1 through Gd-G5 dendrimers extravasated across the BBTB into the extravascular tumor space (Additional file 2; Figure 2C, 2D, and 2E). At the $0.03 \mathrm{mmol} \mathrm{Gd} / \mathrm{kg}$ bw dose, Gd-G6, Gd-G7 and Gd-G8 dendrimers did not extravasate across the BBTB (Figure 2F, 2G, and 2H). At the $0.09 \mathrm{mmol} \mathrm{Gd} / \mathrm{kg}$ bw dose, Gd-G1 through Gd-G6 dendrimers extravasated across the BBTB into the extravascular tumor space (Additional file 2; Figure $2 \mathrm{C}$ through $2 \mathrm{~F}$ ). At the $0.09 \mathrm{mmol} \mathrm{Gd} /$ $\mathrm{kg}$ bw dose, we found that Gd-G7 dendrimers did not extravasate across the less defective BBTB of the smallest gliomas within the size range of brain tumors in our study (Figure 3B). In the case of the largest RG-2 gliomas within the size range of brain tumors in our study, Gd-G7 dendrimers extravasated across the more defective $\mathrm{BBTB}$ as shown in Figure 3A. At both doses, irrespective of the degree of BBTB defectiveness related to tumor size, we found that Gd-G8 dendrimers are impermeable to the BBTB and remain within brain tumor microvasculature (Figure $2 \mathrm{H}$ and Figure 3 ).

\section{Effect of Gd-dendrimer dose and blood half-life on particle} accumulation within brain tumor tissue

At both doses, we found that Gd-G1 through lowly conjugated Gd-G4 dendrimers possess short blood half-lives compared to Gd-dendrimers of higher generations. The blood concentration profile of lowly conjugated Gd-G4 dendrimers was similar to the profiles of Gd-G1, Gd-G2 and Gd-G3 dendrimers suggesting rapid clearance from blood circulation. Standard Gd-G4 dendrimers had a longer blood half-life than lowly conjugated Gd-G4 den- drimers due to the increase in size associated with an approximately $15 \mathrm{kD}$ increase in molecular weight (Figure $2 \mathrm{~A}$ and $2 \mathrm{~B}$, Table 1). At both doses, Gd-G5 through Gd-G8 dendrimers rapidly attained peak blood concentrations and then maintained steady state levels for at least 2 hours following the infusion (Figure 2A and 2B).

At both doses, Gd-G1 through lowly conjugated Gd-G4 dendrimers temporarily accumulated within the extravascular tumor space before wash-out due to short blood half-lives (Additional file 2 and Figure 2C). At both doses, standard Gd-G4 dendrimers remained within the tumor extravascular space longer than the lowly conjugated GdG4 dendrimers (Figure 2D). At both doses, Gd-G5 dendrimers demonstrated a steady rate of accumulation over two hours, although, at the $0.09 \mathrm{mmol} \mathrm{Gd} / \mathrm{kg}$ bw dose the accumulation was faster over the first hour (Figure 2E). At the $0.03 \mathrm{mmol} \mathrm{Gd} / \mathrm{kg}$ bw dose Gd-G6 dendrimers did not accumulate. At the $0.09 \mathrm{mmol} \mathrm{Gd} / \mathrm{kg}$ bw dose, irrespective of tumor size, Gd-G5 and Gd-G6 dendrimers continued to accumulate slowly over 2 hours in all RG-2 gliomas (Figure 2 and Figure 3). Gd-G1 through Gd-G8 dendrimers remained within the microvasculature of normal brain tissue and, as a result, normal brain tissue Gd concentration curves mirrored Gd concentration curves of the superior sagittal sinus (Additional file 3).

\section{Effect of Gd-dendrimer size on transvascular flow rate and particle distribution within brain tumor tissue}

We investigated the relationship between lower Gd-dendrimer generations and tumor volume to the particle transvascular flow rate (permeability, $K^{\text {trans }}$ ) and distribution in the extravascular extracellular tumor volume (fractional extravascular extracellular volume, $v_{\mathrm{e}}$ ) using the 2- 

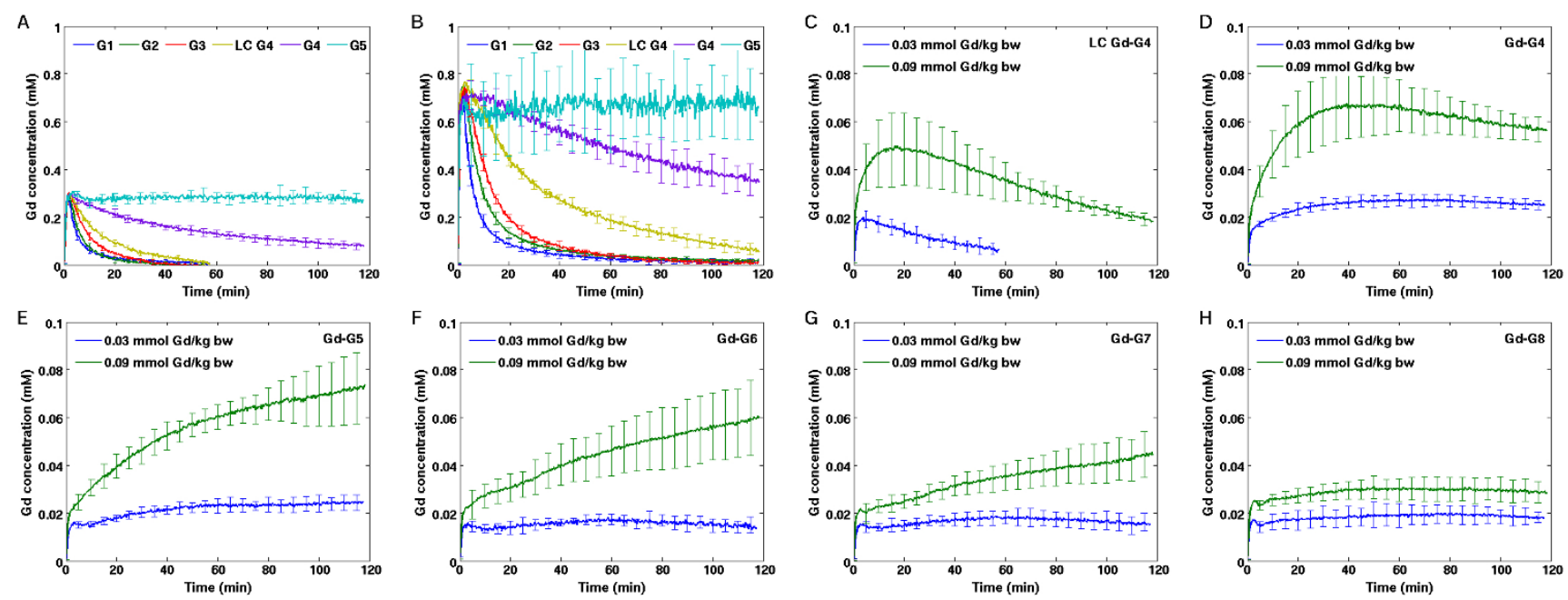

Figure 2

Gd concentration within blood and glioma tissue over time following intravenous Gd-dendrimer infusions at doses of $0.03 \mathrm{mmol} \mathrm{Gd} / \mathbf{k g}$ bw and $0.09 \mathrm{mmol} \mathrm{Gd} / \mathbf{k g ~ b w}$. A) Blood concentrations of Gd-dendrimers measured in the superior sagittal sinus following $0.03 \mathrm{mmol} \mathrm{Gd} / \mathrm{kg}$ bw infusion. Gd-GI (n=6), Gd-G2 (n=5), Gd-G3 ( $=5$ ), and lowly conjugated Gd-G4 (n=5) dendirmers imaged for I hour. Standard Gd-G4 (n=6), Gd-G5 (n=6), Gd-G6 (n=5), Gd-G7 (n=6), and Gd-G8 $(n=5)$ dendrimers imaged for 2 hours. Error bars represent standard deviations. B) Blood concentrations of Gd-dendrimers measured in the superior sagittal sinus following $0.09 \mathrm{mmol} \mathrm{Gd} / \mathrm{kg}$ bw infusion. Gd-GI $(n=4), \mathrm{Gd}-\mathrm{G} 2(\mathrm{n}=6), \mathrm{Gd}-\mathrm{G} 3(\mathrm{n}=6)$, lowly conjugated Gd-G4 (n=4), standard Gd-G4 (n=6), Gd-G5 (n=6), Gd-G6 (n=5), Gd-G7 (n=5), and Gd-G8 (n=6). Blood concentrations of Gd-G6, Gd-G7, and Gd-G8 dendrimers not shown for clarity. C) At both doses, lowly conjugated Gd-G4 dendrimers (molecular weight $24.4 \mathrm{kD}$ ) remain for a short period of time within the extravascular tumor space. $0.03 \mathrm{mmol} \mathrm{Gd} /$ $\mathrm{kg}$ bw dose $\mathrm{n}=5,0.09 \mathrm{mmol} \mathrm{Gd} / \mathrm{kg}$ bw dose $\mathrm{n}=4$. D) At both doses, standard Gd-G4 dendrimers (molecular weight $39.8 \mathrm{kD}$ ) remain for longer within the extravascular tumor space. $0.03 \mathrm{mmol} \mathrm{Gd} / \mathrm{kg}$ bw dose $\mathrm{n}=6,0.09 \mathrm{mmol} \mathrm{Gd} / \mathrm{kg} \mathrm{bw} \mathrm{dose} \mathrm{n}=6$. E) At both doses, Gd-G5 dendrimers accumulate within the extravascular tumor space. $0.03 \mathrm{mmol} \mathrm{Gd} / \mathrm{kg}$ bw dose $\mathrm{n}=6,0.09 \mathrm{mmol}$ $\mathrm{Gd} / \mathrm{kg}$ bw dose $\mathrm{n}=6$. F) At the $0.03 \mathrm{mmol} \mathrm{Gd} / \mathrm{kg}$ bw dose $(\mathrm{n}=5)$, Gd-G6 dendrimers do not extravasate out of tumor microvasculature. At the $0.09 \mathrm{mmol} \mathrm{Gd} / \mathrm{kg}$ bw dose $(\mathrm{n}=5), \mathrm{Gd}-\mathrm{G} 6$ dendrimers extravasate. $\mathrm{G})$ At the $0.03 \mathrm{mmol} \mathrm{Gd} / \mathrm{kg}$ bw dose $(\mathrm{n}=6)$, Gd-G7 dendrimers do not extravasate. At the $0.09 \mathrm{mmol} \mathrm{Gd} / \mathrm{kg}$ bw dose $(\mathrm{n}=5)$, Gd-G7 dendrimers extravasate. H) Irrespective of dose, Gd-G8 dendrimers do not extravasate out of brain tumor microvasculature. $0.03 \mathrm{mmol} \mathrm{Gd} / \mathrm{kg}$ bw dose n=5, 0.09 $\mathrm{mmol} \mathrm{Gd/kg}$ bw dose $n=6$. In panels $\mathrm{C}$ through $\mathrm{H}$, Gd tumor concentrations and standard deviations shown are weighted for total tumor volume.

compartment 3-parameter generalized kinetic model. The third calculated vascular parameter was the tumor fractional plasma volume $\left(v_{\mathrm{p}}\right)[40,50]$. We were able to successfully model the blood and tissue pharmacokinetic behavior of only Gd-G1 through lowly conjugated Gd-G4 dendrimers since these lower Gd-dendrimer generations possess short blood half-lives and, therefore, remain predominantly within the extracellular tumor space. Higher Gd-dendrimer generations do not remain in the extracellular tumor space, but instead accumulate within glioma cells, defying the fundamental assumption of dynamic contrast-enhanced MRI-based modeling that an agent remain extracellular [40].

Based on the range of tumor sizes within the Gd-G1 through lowly conjugated Gd-G4 dendrimer groups, RG2 gliomas were classified as large $\left(>50 \mathrm{~mm}^{3}\right)$ and small $(<$ $50 \mathrm{~mm}^{3}$ ). Irrespective of tumor size, we found significant differences between the four dendrimer generations with respect to particle transvascular flow rates $\left(\mathrm{F}_{3,15.7}=11.61\right.$; Bonferroni corrected $\mathrm{p}=0.0009$, MANOVA) and distribution within the extravascular extracellular tumor volume $\left(\mathrm{F}_{3,16.1}=8.26\right.$; Bonferroni corrected $\mathrm{p}=0.0045$, MANOVA), but not the tumor fractional plasma volume $\left(\mathrm{F}_{3,16.3}=1.24 ; P=\mathrm{NS}, \mathrm{MANOVA}\right)$ (Figure 4A, 4B, and $4 \mathrm{C}$ ). The transvascular flow rate of lowly conjugated Gd-G4 dendrimers was significantly lower compared to that of Gd-G1 dendrimers. As a consequence, lowly conjugated Gd-G4 dendrimers were focally distributed within the extravascular extracellular tumor volume (Figure 4A, 4B, and $4 \mathrm{D}$ ). The vascular plasma volume was not significantly different between tumor populations within the four different dendrimer generations (Figure 4C). Irrespective of dendrimer generation, we found that large tumors had higher values of transvascular flow rates $\left(\mathrm{F}_{1,34.6}=10.83\right.$; Bonferroni corrected $\mathrm{p}=0.0069$, MANOVA), fractional extravascular extracellular volume $\left(\mathrm{F}_{1,22.5}=50.76 ;\right.$ Bonferroni corrected $\mathrm{p}<$ 0.0003, 

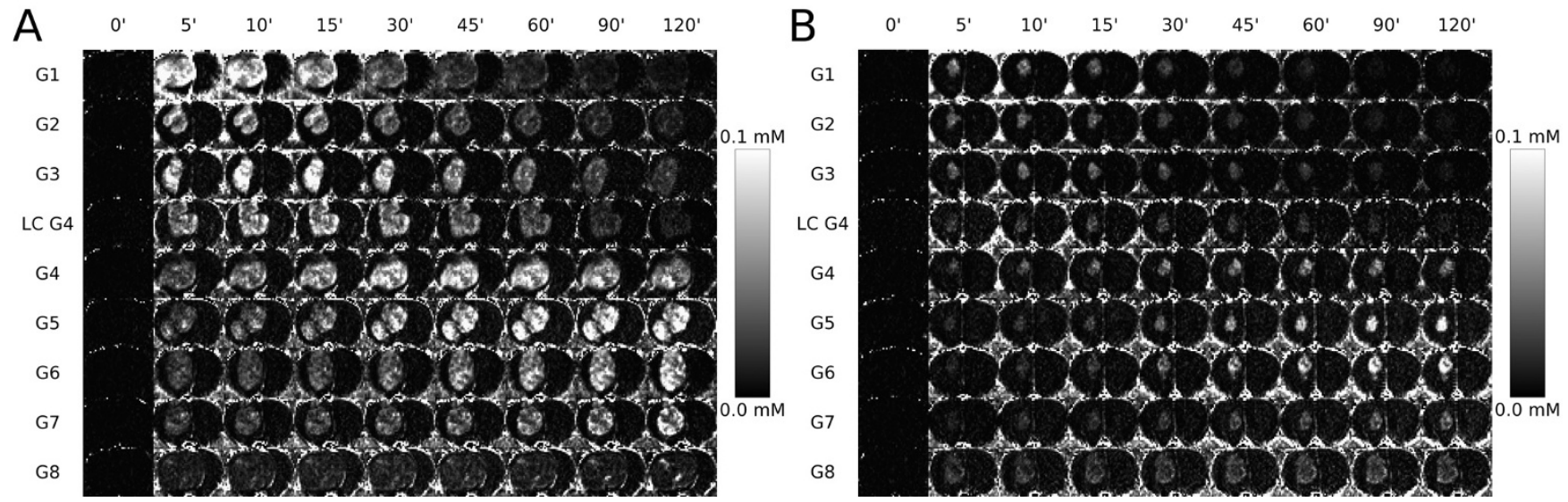

Figure 3

Gd concentration maps showing Gd-dendrimer distribution within the largest and smallest gliomas of each generation over time. A) Gd-G5, Gd-G6, and Gd-G7 dendrimers slowly accumulate within the extravascular tumor space of the largest RG-2 gliomas within the size range of tumors in the study. Gd-G8 dendrimers remain intravascular. The volume, in $\mathrm{mm}^{3}$, for each tumor shown is 104 (Gd-GI), 94 (Gd-G2), 94 (Gd-G3), 162 (lowly conjugated Gd-G4), 200 (standard GdG4), 230 (Gd-G5), 201 (Gd-G6), 170 (Gd-G7), and 289 (Gd-G8). B) Gd-G5 and G6 dendrimers still slowly accumulate within tumor tissue of the smallest RG-2 gliomas, which have a minimally compromised blood-brain tumor barrier. Gd-G7 dendrimers are impermeable to the BBTB of the smallest RG-2 gliomas and remain intravascular. Gd-G8 dendrimers continue to be impermeable to the blood-brain tumor barrier of the smallest RG-2 gliomas. The volume, in $\mathrm{mm}^{3}$, for each tumor shown is 27 (Gd-GI), 28 (Gd-G2), 19 (Gd-G3), 24 (lowly conjugated Gd-G4), 17 (standard Gd-G4), 18 (Gd-G5), 22 (Gd-G6), 24 (Gd-G6), and 107 (Gd-G8). Each animal received an intravenous $0.09 \mathrm{mmol} \mathrm{Gd} / \mathrm{kg}$ bw.
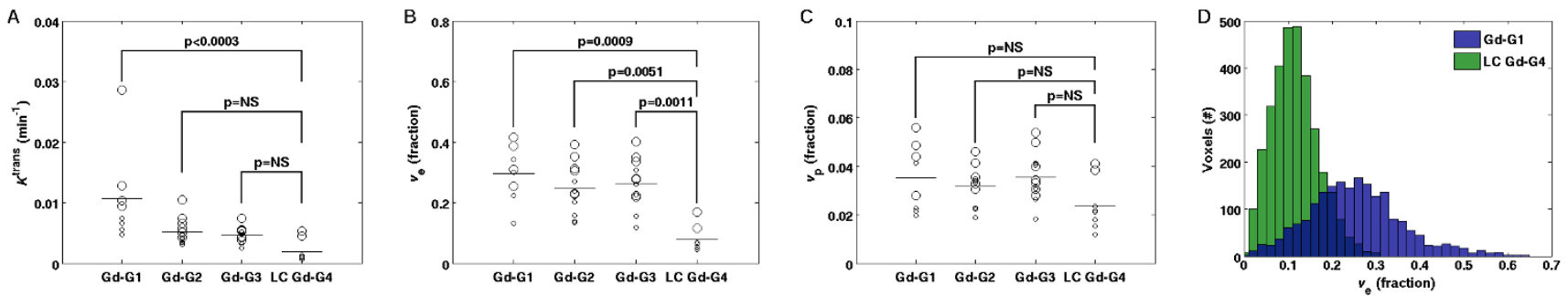

\section{Figure 4}

Modeled pharmacokinetic parameters of lower generation Gd-dendrimers. A) The increase in Gd-dendrimer generation and size from that of Gd-GI to that of lowly conjugated Gd-G4 results in a decrease in particle transvascular flow rate

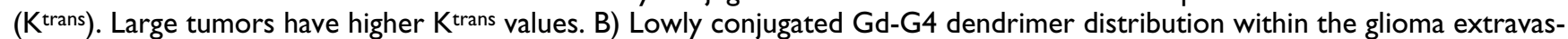
cular extracellular space $\left(\mathrm{v}_{\mathrm{e}}\right)$ is influenced to the greatest extent by the decrease in $\mathrm{K}$ trans. Large tumors have higher $\mathrm{v}_{\mathrm{e}}$ values. C) Fractional plasma volume $\left(v_{p}\right)$ within glioma vasculature is maintained across dendrimer generations. Large tumors have higher $v_{p}$ values. Large circles (Gd-GI $n=4$, Gd-G2 n=6, Gd-G3 n=7, and Gd-G4 n=2) represent large tumors (> $\left.50 \mathrm{~mm}^{3}\right)$, small circles (Gd-GI n=4, Gd-G2 n=6, Gd-G3 n=5, and Gd-G4n=6) represent small tumors $\left(<50 \mathrm{~mm}^{3}\right)$, horizontal bars represent mean of observations weighted with respect to individual tumor volumes. Shown are Bonferroni corrected $p$-values from the nine post hoc comparisons for the three parameters, NS = not significant. D) There a more widespread distribution of Gd-GI particles within the extravascular extracellular tumor space as shown by the greater range of $v_{e}$ values; whereas, there is a more focal distribution of lowly conjugated Gd-G4 dendrimers as shown by the lower range of $v_{e}$ values. Shown are voxels surviving censorship. Tumor volumes, in mm3, for tumors shown are 104 (Gd-GI) and I62 (lowly conjugated Gd-G4). 


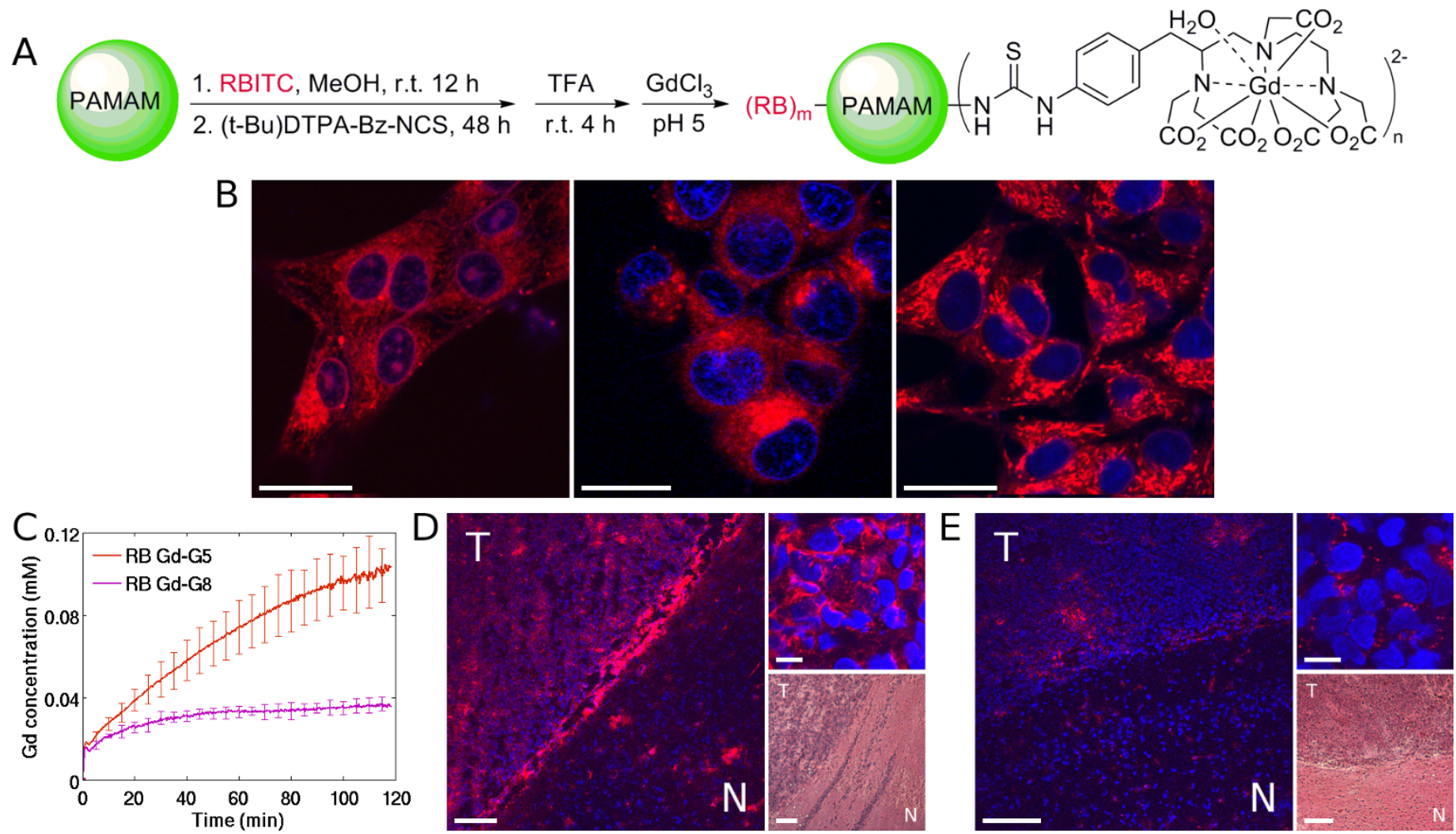

\section{Figure 5}

Fluorescence microscopy of glioma cell uptake of rhodamine B labeled Gd-dendrimer generations in vivo versus ex vivo. A) Synthetic scheme for production of rhodamine B (RB) labeled Gd-polyamidoamine dendrimers. The naked polyamidoamine dendrimer is first reacted with rhodamine $B$ and then with Gd-DTPA. B) As shown by fluorescence microscopy in vitro, rhodamine B Gd-G2, rhodamine B Gd-G5, and rhodamine B Gd-G8 accumulate in glioma cells. Rhodamine B GdG2 dendrimers enter RG-2 glioma cells, and in some cases, the nucleus (left). Rhodamine B Gd-G5 dendrimers enter the cytoplasm of RG-2 glioma cells, but do not localize within the nucleus (middle). Rhodamine B Gd-G8 dendrimers enter RG-2 glioma cells in vitro (right). Shown are merged confocal images of blue fluorescence from DAPI-Vectashield nuclear (DNA) stain and red fluorescence from rhodamine B labeled Gd-dendrimers. Scale bars $=20 \mu \mathrm{m}$. C) At 2 hours dynamic contrast-enhanced MRI shows substantial extravasation of rhodamine B Gd-G5 dendrimers and some extravasation of rhodamine B Gd-G8 dendrimers. Rhodamine $B$ Gd-G5 n=6, rhodamine B Gd-G8 $n=2$. D) Low power fluorescence microscopy ex vivo of brain tumor and normal brain surrounding tumor shows that there is substantial accumulation of rhodamine B Gd-G5 dendrimers within tumor tissue (left, $\mathrm{T}=$ tumor, $\mathrm{N}=$ normal, scale bar $=100 \mu \mathrm{m}$ ). High power shows subcellular localization within malignant glioma cells (upper right, scale bar $=20 \mu \mathrm{m}$ ). Hemotoxylin and Eosin stain of tumor and surrounding brain (lower right, scale bar $=100 \mu \mathrm{m})$. Tumor volume is $31 \mathrm{~mm}^{3}$. E) Also shown by low power fluorescence microscopy ex vivo is some accumulation of rhodamine B Gd-G8 dendrimers within brain tumor tissue (left, $\mathrm{T}=$ tumor, $\mathrm{N}=$ normal, scale bar $=100 \mu \mathrm{m}$ ). High power confirms minimal subcellular localization within glioma cells (upper right, scale bar $=20 \mu \mathrm{m}$ ). Hematoxylin and Eosin stain of tumor and surrounding brain (lower right, scale bar $=100 \mu \mathrm{m}$ ). Tumor volume is $30 \mathrm{~mm}^{3}$.

MANOVA $)$ and fractional plasma volume $\left(\mathrm{F}_{1,27.9}=20.49\right.$; Bonferroni corrected $\mathrm{p}=0.0003, \mathrm{MANOVA})$ than small tumors.

\section{Glioma cell uptake of fluorescent Gd-dendrimer generations in vivo versus ex vivo}

We performed fluorescence microscopy experiments in vitro to confirm that the limitation to particle entry into glioma cells is not at the cellular level. Rhodamine $\mathrm{B}$ labeled Gd-G2, rhodamine B labeled Gd-G5, and rhodamine B labeled Gd-G8 dendrimers were synthesized as representative examples of the Gd-G1 through Gd-G8 dendrimer series. The synthetic scheme of rhodamine $\mathrm{B}$ Gd-dendrimers is shown in Figure 5A. The physical properties of rhodamine B Gd-G2, rhodamine B Gd-G5 and rhodamine B Gd-G8 dendrimers are displayed in Additional file 4 . The physical properties of the rhodamine $\mathrm{B}$ dendrimers were similar to those of the Gd-G2, Gd-G5, and Gd-G8 dendrimers. RG-2 glioma cells were imaged 4 hours after addition of rhodamine B Gd-G2, rhodamine B Gd-G5 or rhodamine B Gd-G8 dendrimers into the culture media at equimolar concentrations with respect to 
rhodamine B. All three Gd-dendrimer generations accumulated within RG-2 glioma cells (Figure 5B). In addition, rhodamine B Gd-G2 dendrimers in some cases were observed to localize within cell nuclei (Figure 5B, left). Rhodamine B Gd-G8 dendrimers localize within glioma cells as readily as rhodamine B Gd-G5 dendrimers indicating that cellular uptake was not the barrier to the accumulation of higher generation Gd-dendrimers within glioma cells.

We conducted additional dynamic contrast-enhanced MRI experiments with correlative fluorescence microscopy of glioma specimens ex vivo to confirm that permeable functionalized dendrimers with long blood half-lives accumulate in glioma cells. The infusion dose for rhodamine B Gd-G5 and rhodamine B Gd-G8 dendrimers was $0.06 \mathrm{mmol} \mathrm{Gd} / \mathrm{kg}$ bw. Rhodamine B labeling of Gd-G5 dendrimers resulted in the enhanced extravasation of rhodamine B Gd-G5 dendrimers across the BBTB and rhodamine B labeling of Gd-G8 dendrimers resulted in some extravasation of rhodamine B Gd-G8 dendrimers across the BBTB, as shown by the dynamic contrastenhanced MRI concentration curves in Figure 5C. There was substantial accumulation of rhodamine B Gd-G5 dendrimers within tumor tissue cells as shown by fluorescence microscopy ex vivo (low power, Figure 5D, left). The subcellular localization of rhodamine B Gd-G5 dendrimers in tumor tissue was similar to what was observed in cultured RG-2 glioma cells (high power, Figure 5D, top right). There was some accumulation of rhodamine B GdG8 dendrimers within tumor tissue (Figure 5E, left). The subcellular localization of rhodamine Gd-G5 dendrimers in tumor tissue was minimal to what was observed in cultured glioma cells (Figure 5E, top right). There was a small amount of extravasation of rhodamine B Gd-G5 and rhodamine B Gd-G8 dendrimer across the normal bloodbrain barrier beginning approximately 1 hour following intravenous infusion, as shown by dynamic contrastenhanced MRI in Additional file 5.

\section{Discussion}

Effective transvascular delivery of therapeutics into malignant glioma cells remains challenging. Although conventional low-molecular weight chemotherapeutics can easily cross the pores within the BBTB of malignant gliomas [21,54], these drugs do not achieve and maintain effective steady state concentrations within malignant glioma cells because of short blood half-lives.

Ultrastructural studies of brain tumor microvasculature have shown that fenestrations and gaps exist within the BBTB ranging from 40 to $90 \mathrm{~nm}$ and 100 to $250 \mathrm{~nm}$, respectively $[20,55]$. Using intravital microscopy, Hobbs et al. [26] have reported that there is primarily perivascular fluorescence in xenografted human malignant gliomas
24 hours after the intravenous infusion of long-circulating rhodamine labeled liposomes $100 \mathrm{~nm}$ in diameter. Using MRI, Moore et al. [25] and Muldoon et al. [56] have reported that there is minimal contrast enhancement of rodent gliomas $24 \mathrm{hrs}$ after the intravenous infusion of various long-circulating dextran coated iron oxide (also known as LCDIO) nanoparticles with a mean diameter of $20 \mathrm{~nm}[57,58]$. These findings indicate that the therapeutically relevant upper limit of the BBTB pore size should range between $20 \mathrm{~nm}$ and $100 \mathrm{~nm}$. However, the effective transvascular delivery of nanoparticle-based drug carriers across the BВTB into malignant glioma cells has remained elusive, to date. We reasoned that the physiologic upper limit of BBTB pores size would be less than $20 \mathrm{~nm}$ in diameter. We were aware that PAMAM dendrimers are particularly small multigenerational nanoparticles of uniform sizes within a generation [31,37]. Functionalized PAMAM dendrimer particle sizes typically range between $1.5 \mathrm{~nm}$ (G1) and $14 \mathrm{~nm}(\mathrm{G} 8)$ in diameter following the conjugation of low molecular weight imaging compounds to the dendrimer exterior [33]. In order to probe the physiologic upper limit of BBTB pore size in RG-2 malignant glioma microvasculature with dynamic contrast-enhanced MRI, we functionalized PAMAM dendrimers G1 through G8 with Gd-DTPA (charge -2) [33,34,45]. As a result of the conjugation of Gd-DTPA to approximately half of the surface amine groups, the positive surface charge on the PAMAM dendrimer exterior was neutralized. In order to confirm that the barrier to cellular entry of Gd-dendrimers is at the level of the BBTB, and that permeable functionalized dendrimers with long blood half-lives can accumulate in malignant glioma cells, we used rhodamine B labeled Gd-dendrimers for fluorescence imaging in vitro and ex vivo. Based on these studies, we report here that the physiologic upper limit of BBTB pore size ranges between approximately 11.7 and $11.9 \mathrm{~nm}$. We also report that permeable functionalized dendrimers with long blood half-lives can accumulate within glioma cells.

We observed that there was virtually no contrast enhancement of malignant glioma tissue over 2 hours on dynamic-contrast enhanced MRI following the intravenous infusion of Gd-G8 dendrimers. We found this to be the case at both $\mathrm{Gd}$-dendrimer doses investigated, one being the standard $0.03 \mathrm{mmol} \mathrm{Gd} / \mathrm{kg}$ bw dose for pre-clinical dynamic contrast-enhanced MRI and the other being $0.09 \mathrm{mmol} \mathrm{Gd} / \mathrm{kg}$ bw [33]. These dynamic contrastenhanced MRI findings demonstrate that Gd-G8 dendrimers are larger than the upper limit of the physiologic pore size of the BBTB of RG-2 gliomas. Using ADF STEM, we measured the diameters of a population of our Gd-G8 dendrimers to be $13.3 \pm 1.4 \mathrm{~nm}$ (mean \pm standard deviation) and that of Gd-G7 dendrimers to be $11.0 \pm 0.7 \mathrm{~nm}$. Based on these ADF STEM data, the range of the physio- 
logic upper limit of BBTB pore size in RG-2 malignant gliomas is between 11.7 and $11.9 \mathrm{~nm}$.

To confirm that the limitation to functionalized G8 dendrimer entry is not at the cellular level, we performed fluorescence microscopy of cultured RG-2 glioma cells following the application of rhodamine B labeled Gddendrimers to the media. We found that rhodamine $\mathrm{B}$ labeled Gd-G2, -G5 and -G8 dendrimers accumulated in the cytoplasm of all RG-2 glioma cells; however, we found it particularly interesting that, in some cases, rhodamine B labeled Gd-G2 dendrimers also accumulated in the RG-2 glioma cell nuclei. This finding suggests that it may also be possible for other smaller nanoparticles (i.e. molecular weight $\leq 11.2 \mathrm{kD}$ ) to cross nuclear pores.

Irrespective of dose, we found that Gd-G1, Gd-G2, Gd-G3 and lowly conjugated Gd-G4 (molecular weight $24.4 \mathrm{kD}$ ) dendrimers had short blood half-lives because particle sizes of these lower generation Gd-dendrimers are small enough that particles can be efficiently filtered by the kidneys [17]. Therefore, Gd-G1 through lowly conjugated Gd-G4 dendrimers only remain temporarily within the tumor extravascular extracellular space. We also found that as the Gd-dendrimer generation and particle size increased, the transvascular flow (Krans) rate decreased; and that the lower transvascular flow rate of lowly conjugated Gd-G4 dendrimers resulted in the more focal distribution of particles within brain tumor tissue. Therefore, since lower generation dendrimers have short blood halflives, the transvascular flow rate across the BBTB is the primary determinant of how widespread particle distribution was within the extravascular extracellular tumor space. These findings suggest that nanoparticles with higher molecular weights, yet particle sizes small enough to still be effectively filtered by the kidneys, do not remain within the extravascular tumor space sufficiently long to effectively permeate through tumor tissue. Therefore, such nanoparticles would remain within close proximity of tumor microvessels, and would not reach malignant glioma cells located within tumor regions that are poorly vascularized.

We found that standard Gd-G4 dendrimers (molecular weight $39.8 \mathrm{kD}$ ) had a longer blood half-life than the lower generation Gd-dendrimers because the particle size of standard Gd-G4 dendrimers is at the threshold of effective renal filtration [17]. Irrespective of dose, Gd-G5 through Gd-G8 dendrimers maintained steady state blood concentrations over a minimum of 2 hours because particle sizes of these generations of Gd-dendrimers are clearly above the threshold of effective renal filtration [17]. As a result of the long blood half-lives, Gd-G5 and Gd-G6 were able to slowly extravasate across the BBTB of even the smallest gliomas that we studied. Based on these findings, we conclude that it may be possible to effectively deliver permeable nanoparticles with long blood halflives across a minimally compromised BBTB, including across the BBTB of the microvasculature supplying emerging malignant glioma colonies.

To verify that only permeable functionalized dendrimers with long blood half-lives accumulate within malignant glioma cells, we infused rhodamine B labeled Gd-G5 dendrimers and rhodamine B labeled Gd-G8 dendrimers to separate groups of rats. The dose of rhodamine B Gd-dendrimers was $0.06 \mathrm{mmol} \mathrm{Gd} / \mathrm{kg} \mathrm{bw}$, since in pilot experiments we observed that the anesthetic effect of isoflurane was potentiated at the $0.09 \mathrm{mmol} \mathrm{Gd} / \mathrm{kg}$ bw rhodamine $\mathrm{B}$ Gd-dendrimer dose $[59,60]$. Fluorescence microscopy of RG-2 glioma specimens demonstrated extensive subcellular localization of rhodamine B Gd-G5 dendrimers, confirming that functionalized G5 dendrimers accumulate within malignant glioma cells, due to long blood halflives.

We observed with both fluorescence microscopy and dynamic contrast-enhanced MRI that there was some accumulation of rhodamine B Gd-G8 dendrimers in RG-2 gliomas (Figure 5C and 5E), as well as some non-selective accumulation of rhodamine B Gd-G5 and rhodamine B Gd-G8 dendrimers in tumor-free brain regions (Additional file 5). We suspect that rhodamine B labeled Gd-G5 and Gd-G8 dendrimers are toxic to the BBTB in addition to the otherwise healthy blood-brain barrier. This toxicity is likely due to the introduction of additional positive charge to the Gd-dendrimer surface from the attachment of rhodamine $\mathrm{B}$, a cationic and lipophilic fluorescent dye [61-64]. Therefore, the extravasation of rhodamine labeled nanoparticles $[26,65]$ and other charged nanoparticles [66-69] across the barrier may be from direct charge induced damage to endothelial cells of the barrier and disruption of the barrier. Our proposed mechanism for the increased barrier permeation of rhodamine labeled Gddendrimers is analogous to the mechanism recently proposed by Herce and Garcia $[70,71]$ for the movement of cell-penetrating peptides across cell membranes. We plan to clarify, in the future, with additional in vivo imaging experiments, the relationship between charge on the dendrimer surface and disruption of the blood-brain barrier.

\section{Conclusion}

In this study, we identified the precise physiologic upper limit of blood-brain tumor barrier pore size, and demonstrated that nanoparticles of diameters smaller than this upper limit can effectively traverse the pores of the bloodbrain tumor barrier; in addition, we validated the importance of prolonged nanoparticle blood half-life for the effective accumulation of nanoparticles within brain tumor cells. Therefore, based on these findings, we con- 
clude that effective drug delivery across the BBTB of malignant gliomas, and potentially the BBB of other neuropathologies, can be accomplished with non-toxic nanoparticles that are smaller than 11.7 to $11.9 \mathrm{~nm}$ in diameter and have prolonged blood half-lives.

In the broadest sense, our findings will serve as general guidelines, for the future design and development of multifunctional transvascular delivery devices, based on nanoparticles (i.e. liposome-, quantum dot-, or iron oxidebased) and biological particles (i.e. antibody- or viralbased), that are particularly effective at crossing the diseased BBB and accumulating in neuropathologic tissues.

\section{Competing interests}

The authors declare that they have no competing interests.

\section{Authors' contributions}

HS conceptualized, designed, and supervised the overall study; performed the dynamic contrast-enhanced MRI experiments, analyzed the data, interpreted the overall study results, and prepared the manuscript. ASK performed the dynamic contrast-enhanced MRI experiments, analyzed the data, and assisted with the preparation of the manuscript. HW synthesized and performed the preliminary characterization of the functionalized dendrimers. $\mathrm{KRB}$ assisted with the confocal fluorescence microscopy experiments. SHF performed the initial dynamic contrastenhanced MRI experiments. KS assisted with the preparation of the manuscript. AAS characterized the higher generation functionalized dendrimers by electron microscopy. SA performed the statistical data analysis. CMW assisted with the synthesis of the functionalized dendrimers. MAA assisted with the characterization of the higher generation functionalized dendrimers by electron microscopy. RDL supervised the electron microscopybased characterization of the functionalized dendrimers. GLG supervised the synthesis and preliminary characterization of the functionalized dendrimers, and contributed to the design of the overall study. MDH conceptualized, designed, and supervised the confocal fluorescence microscopy experiments; assisted with the interpretation of the overall study results, and prepared the manuscript.

\section{Additional material}

\section{Additional file 1}

Amount of Gd-PAMAM dendrimer infused per Gd dose.

Click here for file

[http://www.biomedcentral.com/content/supplementary/1479-

5876-6-80-S1.pdf]

\section{Additional file 2}

Gd-dendrimer residence time within the extravascular extracellular brain tumor space increases with increasing dendrimer generation at $0.09 \mathrm{mmol} \mathrm{Gd} / \mathrm{kg}$ body weight dose. At the $0.03 \mathrm{mmol} \mathrm{Gd} / \mathrm{kg}$ bw dose, changes in the concentration profiles of Gd-G1 (left), Gd-G2 (middle) and Gd-G3 (right) are not evident. $0.09 \mathrm{mmol} \mathrm{Gd} / \mathrm{kg}$ body weight dose, Gd-G1 $(n=5), G d-G 2(n=6), G d-G 3(n=6) .0 .03 \mathrm{mmol} \mathrm{Gd} / \mathrm{kg} b w$ dose, Gd-G1 $(n=6), G d-G 2(n=5), G d-G 3(n=5)$. Error bars represent standard deviation weighted for total tumor volume and are shown once every five minutes for clarity. Average tumor concentration curves are weighted with respect to total tumor volume within the respective dendrimer generation.

Click here for file

[http://www.biomedcentral.com/content/supplementary/14795876-6-80-S2.jpeg]

\section{Additional file 3}

Gd-dendrimers do not enter the normal brain extravascular space due to the normal blood-brain barrier. Shown are dynamic contrastenhanced MRI concentration curves at the $0.09 \mathrm{mmol} \mathrm{Gd} / \mathrm{kg}$ body weight dose. Gd-G1 $(n=5)$ and Gd-G5 $(n=6)$ as representative examples of low and high dendrimer generation behavior. Error bars represent standard deviation and are shown once every five minutes for clarity. Average concentration curves are from normal brain tissue volumes of $9 \mathrm{~mm}^{3} \mathrm{per}$ brain.

Click here for file

[http://www.biomedcentral.com/content/supplementary/1479-

5876-6-80-S3.jpeg]

\section{Additional file 4}

Physical properties of rhodamine B Gd-PAMAM dendrimers.

Click here for file

[http://www.biomedcentral.com/content/supplementary/1479-

5876-6-80-S4.pdf]

\section{Additional file 5}

Rhodamine labeled $\mathrm{Gd}-\mathrm{G} 5$ and rhodamine labeled $\mathrm{Gd}-\mathrm{G} 8$ dendrimers enter the normal brain extravascular space across the normal bloodbrain barrier. Shown are dynamic contrast-enhanced MRI concentration curves of rhodamine Gd-dendrimers at a $0.06 \mathrm{mmol} \mathrm{Gd} / \mathrm{kg}$ body weight dose and $\mathrm{Gd}$-dendrimers at a $0.09 \mathrm{mmol} \mathrm{Gd} / \mathrm{kg}$ body weight dose. A) Rhodamine Gd-G5 $(n=6), G d-G 5(n=6)$. B) Rhodamine Gd-G8 $(n=$ 2), Gd-G8 $(n=6)$. Error bars represent standard deviation and are shown once every five minutes for clarity. Average concentration curves are from normal brain tissue volumes of $9 \mathrm{~mm}^{3}$ per brain.

Click here for file

[http://www.biomedcentral.com/content/supplementary/1479-

5876-6-80-S5.jpeg]

\section{Acknowledgements}

This study was funded by the National Institute of Biomedical Imaging Bioengineering (NIBIB), National Cancer Institute ( $\mathrm{NCl}$ ), and the Radiology and Imaging Sciences Program (CC). We thank Guofeng Zhang of the Laboratory of Bioengineering and Physical Science (NIBIB) and Yide Mi of the Radiology and Imaging Sciences Program (CC) for technical assistance. We thank Daniel Glen and Rick Reynolds of the Scientific and Statistical Computing Core (National Institute of Mental Health [NIMH]) for their assistance during our use of the Analysis of Functional Neurolmages (AFNI) software suite for data analyses. 


\section{References}

I. Weber WA, Czernin J, Phelps ME, Herschman HR: Technology Insight: novel imaging of molecular targets is an emerging area crucial to the development of targeted drugs. Nat Clin Pract Oncol 2008, 5:44-54.

2. Wolinsky JB, Grinstaff MW: Therapeutic and diagnostic applications of dendrimers for cancer treatment. Adv Drug Deliv Rev 2008, 60:1037-1055

3. Neuwelt E, Abbott NJ, Abrey L, Banks WA, Blakley B, Davis T, Engelhardt B, Grammas P, Nedergaard M, Nutt J, et al.: Strategies to advance translational research into brain barriers. Lancet Neurol 2008, 7:84-96.

4. Walker MD, Green SB, Byar DP: Randomized comparisons of radiotherapy and nitrosoureas for the treatment of malignant glioma after surgery. New England Journal of Medicine 1980, 303: I323-1329.

5. Stupp R, Mason WP, Bent MJ van den, Weller M, Fisher B, Taphoorn MJ, Belanger K, Brandes AA, Marosi C, Bogdahn U, et al.: Radiotherapy plus concomitant and adjuvant temozolomide for glioblastoma. N Engl J Med 2005, 352:987-996.

6. Lin SH, Kleinberg LR: Carmustine wafers: localized delivery of chemotherapeutic agents in CNS malignancies. Expert Rev Anticancer Ther 2008, 8:343-359.

7. Cohen MH, Johnson JR, Pazdur R: Food and Drug Administration Drug approval summary: temozolomide plus radiation therapy for the treatment of newly diagnosed glioblastoma multiforme. Clin Cancer Res 2005, I I:6767-677I.

8. Brem H, Mahaley MS Jr, Vick NA, Black KL, Schold SC Jr, Burger PC, Friedman $\mathrm{AH}$, Ciric IS, Eller TW, Cozzens JW, et al.: Interstitial chemotherapy with drug polymer implants for the treatment of recurrent gliomas. J Neurosurg I991, 74:44|-446.

9. Westphal M, Hilt DC, Bortey E, Delavault P, Olivares R, Warnke PC, Whittle IR, Jaaskelainen J, Ram Z: A phase 3 trial of local chemotherapy with biodegradable carmustine (BCNU) wafers (Gliadel wafers) in patients with primary malignant glioma. Neuro Oncol 2003, 5:79-88.

10. Gallia GL, Brem S, Brem H: Local treatment of malignant brain tumors using implantable chemotherapeutic polymers. J Natl Compr Canc Netw 2005, 3(5):721-728.

II. Fung LK, Ewend MG, Sills A, Sipos EP, Thompson R, Watts M, Colvin OM, Brem H, Saltzman WM: Pharmacokinetics of interstitial delivery of carmustine, 4-hydroperoxycyclophosphamide, and paclitaxel from a biodegradable polymer implant in the monkey brain. Cancer Research 1998, 58:672-684

12. Brem H, Piantadosi S, Burger PC, Walker M, Selker R, Vick NA, Black K, Sisti M, Brem S, Mohr G, et al.: Placebo-controlled trial of safety and efficacy of intraoperative controlled delivery by biodegradable polymers of chemotherapy for recurrent gliomas. The Polymer-brain Tumor Treatment Group. Lancet 1995, 345:1008-1012.

13. Newlands ES, Stevens MF, Wedge SR, Wheelhouse RT, Brock C: Temozolomide: a review of its discovery, chemical properties, pre-clinical development and clinical trials. Cancer Treat Rev 1997, 23:35-6I.

14. Allen TM, Cullis PR: Drug delivery systems: entering the mainstream. Science 2004, 303:1818-1822.

15. Langer R: Drug delivery and targeting. Nature 1998, 392:5-10.

16. Asgeirsson D, Venturoli D, Fries E, Rippe B, Rippe C: Glomerular sieving of three neutral polysaccharides, polyethylene oxide and bikunin in rat. Effects of molecular size and conformation. Acta Physiologica 2007, I 91 :237-246.

17. Soo Choi H, Liu W, Misra P, Tanaka E, Zimmer JP, Itty Ipe B, Bawendi MG, Frangioni JV: Renal clearance of quantum dots. Nat Biotechnol 2007, 25: I 165-1 170.

18. Knauf MJ, Bell DP, Hirtzer P, Luo ZP, Young JD, Katre NV: Relationship of effective molecular size to systemic clearance in rats of recombinant interleukin-2 chemically modified with water-soluble polymers. Journal of Biological Chemistry 1988, 263: I5064-15070.

19. Matsumura $\mathrm{Y}$, Maeda $\mathrm{H}$ : A new concept for macromolecular therapeutics in cancer chemotherapy: mechanism of tumoritropic accumulation of proteins and the antitumor agent smancs. Cancer Res 1986, 46:6387-6392.

20. Vick NA, Bigner DD: Microvascular abnormalities in virallyinduced canine brain tumors. Structural bases for altered blood-brain barrier function. J Neurol Sci 1972, I 7:29-39.
21. Vick NA, Khandekar JD, Bigner DD: Chemotherapy of brain tumors. The "blood-brain barrier" is not a factor. Arch Neurol 1977, 34:523-526.

22. Brem H: Polymers to treat brain tumours. Biomaterials 1990 , I I:699-70I.

23. Siegal T, Horowitz A, Gabizon A: Doxorubicin encapsulated in sterically stabilized liposomes for the treatment of a brain tumor model: biodistribution and therapeutic efficacy. Genomics \& Informatics 2006, 4(4 | 47- 60 [http://www.genominfo.org/ html/UploadFile/article2 2006l2.pdf].

24. Brigger I, Morizet J, Laudani L, Aubert G, Appel M, Velasco V, TerrierLacombe MJ, Desmaele D, d'Angelo J, Couvreur P, Vassal G: Negative preclinical results with stealth nanospheres-encapsulated Doxorubicin in an orthotopic murine brain tumor model. I Control Release 2004, I 00:29-40.

25. Moore A, Marecos E, Bogdanov A Jr, Weissleder R: Tumoral distribution of long-circulating dextran-coated iron oxide nanoparticles in a rodent model. Radiology 2000, 2 I 4:568-574.

26. Hobbs SK, Monsky WL, Yuan F, Roberts WG, Griffith L, Torchilin VP, Jain RK: Regulation of transport pathways in tumor vessels: role of tumor type and microenvironment. Proc Natl Acad Sci USA 1998, 95:4607-46I2.

27. Chertok B, Moffat BA, David AE, Yu F, Bergemann C, Ross BD, Yang $V C$ : Iron oxide nanoparticles as a drug delivery vehicle for MRI monitored magnetic targeting of brain tumors. Biomaterials 2008, 29:487-496.

28. Fabel K, Dietrich J, Hau P, Wismeth C, Winner B, Przywara S, Steinbrecher A, Ullrich W, Bogdahn U: Long-term stabilization in patients with malignant glioma after treatment with liposomal doxorubicin. Cancer 2001, 92:1936-1942.

29. Wu G, Barth RF, Yang W, Kawabata S, Zhang L, Green-Church K: Targeted delivery of methotrexate to epidermal growth factor receptor-positive brain tumors by means of cetuximab (IMC-C225) dendrimer bioconjugates. Mol Cancer Ther 2006, 5:52-59.

30. Rainov NG, Dobberstein KU, Heidecke V, Dorant U, Chase M, Kramm CM, Chiocca EA, Breakefield XO: Long-term survival in a rodent brain tumor model by bradykinin-enhanced intraarterial delivery of a therapeutic herpes simplex virus vector. Cancer Gene Therapy 1998, 5:158-162.

31. Tomalia DA, Frechet JM: Discovery of dendrimers and dendritic polymers: a brief historical perspective. Journal of Polymer Science, Part A: Polymer Chemistry 2002, 40:2719-2728.

32. Tomalia DA, Reyna LA, Svenson S: Dendrimers as multi-purpose nanodevices for oncology drug delivery and diagnostic imaging. Biochem Soc Trans 2007, 35:61-67.

33. Kobayashi H, Brechbiel MW: Nano-sized MRI contrast agents with dendrimer cores. Adv Drug Deliv Rev 2005, 57:227I-2286.

34. Wiener EC, Brechbiel MW, Brothers H, Magin RL, Gansow OA, Tomalia DA, Lauterbur PC: Dendrimer-based metal chelates: a new class of magnetic resonance imaging contrast agents. Magn Reson Med 1994, 3 I : I-8.

35. Kukowska-Latallo JF, Candido KA, Cao Z, Nigavekar SS, Majoros IJ, Thomas TP, Balogh LP, Khan MK, Baker JR Jr: Nanoparticle targeting of anticancer drug improves therapeutic response in animal model of human epithelial cancer. Cancer Res 2005, 65:5317-5324.

36. Myc A, Douce TB, Ahuja N, Kotlyar A, Kukowska-Latallo J, Thomas TP, Baker JR Jr: Preclinical antitumor efficacy evaluation of dendrimer-based methotrexate conjugates. Anticancer Drugs 2008, 19:143-149.

37. Jackson CL, Chanzy HD, Booy FP, Drake BJ, Tomalia DA, Bauer BJ, Amis EJ: Visualization of dendrimer molecules by transmission electron microscopy (TEM): Staining methods and cryoTEM of vitrified solutions. Macromolecules I998, 3 I:6259-6265.

38. Aas AT, Brun A, Blennow C, Stromblad S, Salford LG: The RG2 rat glioma model. J Neurooncol 1995, 23:175-183.

39. Barth RF: Rat brain tumor models in experimental neurooncology: The 9L, C6, T9, F98, RG2 (D74), RT-2 and CNS-I gliomas. Journal of Neuro-Oncology 1998, 36:91-I02.

40. Tofts PS, Kermode AG: Measurement of the blood-brain barrier permeability and leakage space using dynamic MR imaging. I. Fundamental concepts. Magn Reson Med I99I, 17:357-367.

4I. Ferrier MC, Sarin H, Fung SH, Schatlo B, Pluta RM, Gupta SN, Choyke $\mathrm{PL}$, Oldfield $\mathrm{EH}$, Thomasson D, Butman JA: Validation of dynamic 
contrast-enhanced magnetic resonance imaging-derived vascular permeability measurements using quantitative autoradiography in the RG2 rat brain tumor model. Neoplasia 2007, 9:546-555.

42. Haacke EM, Brown RW, Thompson MR, Venkatesan M: Magnetic Resonance Imaging: Physical Principles and Sequence Design New York: Wiley; 1999.

43. Moore JL, Taylor SM, Soloshonok VA: An efficient and operationally convenient general synthesis of tertiary amines by direct alkylation of secondary amines with alkyl halides in the presence of Huenig's base. Arkivoc 2005, 2005:287-292.

44. Brechbiel MW, Gansow OA, Atcher RW, Schlom J, Esteban J, Simpson DE, Colcher D: Synthesis of I-(p-isothiocyanatobenzyl) derivatives of DTPA and EDTA. Antibody labeling and tumor-imaging studies. Inorganic Chemistry 1986, 25:2772-278I.

45. Xu H, Regino CA, Bernardo M, Koyama Y, Kobayashi H, Choyke PL, Brechbiel MW: Toward improved syntheses of dendrimerbased magnetic resonance imaging contrast agents: new bifunctional diethylenetriaminepentaacetic acid ligands and nonaqueous conjugation chemistry. J Med Chem 2007, 50:3185-3193.

46. Sousa AA, Leapman RD: Quantitative STEM mass measurement of biological macromolecules in a $300 \mathrm{kV}$ TEM. J Microsc 2007, 228:25-33.

47. Paxinos G, Watson C: The Rat Brain in Stereotaxic Coordinates 4th edition. New York: Elsevier; 2004.

48. Cox RW: AFNI: software for analysis and visualization of functional magnetic resonance neuroimages. Comput Biomed Res 1996, 29:162-173.

49. Lee HB, Blaufox MD: Blood volume in the rat. J Nucl Med 1985, 26:72-76.

50. Tofts PS, Brix G, Buckley DL, Evelhoch JL, Henderson E, Knopp MV, Larsson HB, Lee TY, Mayr NA, Parker GJ, et al.: Estimating kinetic parameters from dynamic contrast-enhanced $T(I)$-weighted MRI of a diffusable tracer: standardized quantities and symbols. J Magn Reson Imaging 1999, 10:223-232.

5I. Prosa TJ, Bauer BJ, Amis EJ, Tomalia DA, Scherrenberg R: A SAXS study of the internal structure of dendritic polymer systems. Journal of Polymer Science Part B: Polymer Physics 1997, 35:29|3-2924.

52. Nisato G, Ivkov R, Amis EJ: Size invariance of polyelectrolyte dendrimers. Macromolecules 2000, 33:4172-4I76.

53. Sousa A, Aronova MA, Wu H, Sarin H, Griffiths GL, Leapman RD: Quantitative STEM and EFTEM characterization of dendrimer-based nanoparticles used in magnetic resonance imaging and drug delivery. Microsc Microanal 2008, I 4 Suppl 2:694-695

54. Gerstner ER, Fine RL: Increased permeability of the bloodbrain barrier to chemotherapy in metastatic brain tumors: establishing a treatment paradigm. J Clin Oncol 2007, 25:2306-2312.

55. Schlageter KE, Molnar P, Lapin GD, Groothuis DR: Microvessel organization and structure in experimental brain tumors: Microvessel populations with distinctive structural and functional properties. Microvascular Research 1999, 58:312-328.

56. Muldoon LL, Sandor M, Pinkston KE, Neuwelt EA: Imaging, distribution, and toxicity of superparamagnetic iron oxide magnetic resonance nanoparticles in the rat brain and intracerebral tumor. Neurosurgery 2005, 57:

57. Shen T, Weissleder R, Papisov M, Bogdanov A Jr, Brady TJ: Monocrystalline iron oxide nanocompounds (MION): Physicochemical properties. Magnetic Resonance in Medicine 1993, 29:599-604.

58. Jung CW, Jacobs P: Physical and chemical properties of superparamagnetic iron oxide MR contrast agents: ferumoxides, ferumoxtran, ferumoxsil. Magn Reson Imaging 1995, I 3:66|-674.

59. Summerhayes IC, Lampidis TJ, Bernal SD: Unusual retention of rhodamine 123 by mitochondria in muscle and carcinoma cells. Proceedings of the National Academy of Sciences of the United States of America 1982, 79:5292-5296.

60. Gear ARL: Rhodamine 6G. A potent inhibitor of mitochondrial oxidative phosphorylation. Journal of Biological Chemistry 1974, 249:3628-3637.

61. Malik N, Wiwattanapatapee R, Klopsch R, Lorenz K, Frey H, Weener JW, Meijer EW, Paulus W, Duncan R: Dendrimers: relationship between structure and biocompatibility in vitro, and prelim- inary studies on the biodistribution of | $25 \mid$-labelled polyamidoamine dendrimers in vivo. J Control Release 2000, 65: I33-148.

62. Lutty GA: The acute intravenous toxicity of biological stains, dyes, and other fluorescent substances. Toxicology and Applied Pharmacology 1978, 44:225-249.

63. Ravnic DJ, Zhang YZ, Turhan A, Tsuda A, Pratt JP, Huss HT, Mentzer S): Biological and optical properties of fluorescent nanoparticles developed for intravascular imaging. Microscopy Research and Technique 2007, 70:776-78I.

64. Bingaman S, Huxley VH, Rumbaut RE: Fluorescent dyes modify properties of proteins used in microvascular research. Microcirculation 2003, 10:221-231.

65. Kim JS, Yoon TJ, Yu KN, Kim BG, Park SJ, Kim HW, Lee KH, Park SB, Lee JK, Cho MH: Toxicity and tissue distribution of magnetic nanoparticles in mice. Toxicological Sciences 2006, 89:338-347.

66. Lockman PR, Koziara JM, Mumper RJ, Allen DD: Nanoparticle Surface Charges Alter Blood-Brain Barrier Integrity and Permeability. Journal of Drug Targeting 2004, 12:635-64I.

67. Kang YS, Pardridge WM: Brain delivery of biotin bound to a conjugate of neutral avidin and cationized human albumin. Pharmaceutical Research 1994, I I: 1257- 264.

68. Costantino L, Gandolfi F, Tosi G, Rivasi F, Vandelli MA, Forni F: Peptide-derivatized biodegradable nanoparticles able to cross the blood-brain barrier. Journal of Controlled Release 2005, 108:84-96.

69. Poduslo JF, Curran GL: Polyamine modification increases the permeability of proteins at the blood-nerve and blood-brain barriers. Journal of Neurochemistry 1996, 66:1599-1609.

70. Herce HD, Garcia AE: Molecular dynamics simulations suggest a mechanism for translocation of the HIV-I TAT peptide across lipid membranes. Proceedings of the National Academy of Sciences of the United States of America 2007, I 04:20805-208I0.

7I. Herce HD, Garcia AE: Cell Penetrating Peptides: How Do They Do It? Journal of Biological Physics 2008: I-12.
Publish with Bio Med Central and every scientist can read your work free of charge

"BioMed Central will be the most significant development for disseminating the results of biomedical research in our lifetime. "

Sir Paul Nurse, Cancer Research UK

Your research papers will be:

- available free of charge to the entire biomedical community

- peer reviewed and published immediately upon acceptance

- cited in PubMed and archived on PubMed Central

- yours - you keep the copyright 